\title{
ON REFINED STARK CONJECTURES IN THE NON-ABELIAN CASE
}

\author{
DAVID BURNS
}

\begin{abstract}
We discuss an explicit integral refinement of Stark's Conjecture in the general (non-abelian) case. We show that, upon specialization to the case of odd irreducible degree two complex characters of $\operatorname{Gal}(\overline{\mathbb{Q}} / \mathbb{Q})$ for which the associated $L$-function vanishes to order one at $s=0$, our conjecture refines a question of Stark and a conjecture of Chinburg. As supporting evidence for our conjecture we give a full proof in the function field case and (in the number field case) a proof for rational valued characters and for degree one characters of either $\operatorname{Gal}(\overline{\mathbb{Q}} / \mathbb{Q})$ or $\operatorname{Gal}(\overline{\mathbb{Q}} / k)$ for suitable imaginary quadratic fields $k$.
\end{abstract}

\section{Introduction}

The principal conjecture of Stark, as interpreted by Tate in [21], asserts (roughly speaking) that the leading term at $s=0$ of Artin $L$-functions is equal, to within an undetermined algebraic factor, to a regulator constructed from algebraic units. For Artin $L$-functions associated to characters that factor through abelian Galois extensions, subsequent refinements of this conjecture (due firstly to Stark [19] with later refinements and generalisations by, amongst others, Gross, Tate and Rubin) have had the effect of bounding the denominator of the undetermined algebraic factor. An analogous refinement in the general (non-abelian) case would have significant advantages over the original conjecture of Stark (not least for the purposes of obtaining evidence via computer calculations - see the discussion of Dummit in $[10, \S 14]$ ). Indeed, for the special case of odd irreducible complex representations of $\mathrm{Gal}(\overline{\mathbb{Q}} / \mathbb{Q})$ that are of degree 2 and such that the associated $L$-function vanishes to order 1 at $s=0$, the connection to modular forms provided by the theorem of Deligne and Serre first led Stark [20] to investigate the possibility of an explicit such 'integral' refinement and then Chinburg [8] to formulate a precise conjecture for this special case (see the discussion of [21, Ch. III, §4]). Computer calculations of, amongst others, Chinburg [8], Fogel [12] and Jehanne, Roblot and Sands [14] have since given credence to this conjecture (which is referred to in loc. cit. as the 'Stark-Chinburg Conjecture'). However, it has been a long-standing problem to give a more conceptual approach to Stark's question and to Chinburg's conjecture and perhaps thereby suggest an explicit integral refinement of Stark's Conjecture in a more general setting.

In $\S 2$ of this article we shall now formulate, as Conjecture 2.1, an explicit integral refinement of Stark's Conjecture in the most general possible case (that is, for any non-trivial irreducible complex representation that is associated to any finite Galois extension of any global field). This conjecture is in effect a natural non-abelian

2000 Mathematics Subject Classification. 11S40.

The author was supported by a Leverhulme Research Fellowship 
analogue of the conjecture formulated by Rubin in [18, Conj. B]. Further, in $\S 3$ we show that, upon specialisation to the cases considered by Stark and Chinburg, Conjecture 2.1 refines both the original question posed by Stark in [20] and also (in any case in which the Euler factors of all primes which ramify in the given Galois extension are trivial) the Stark-Chinburg Conjecture itself. At the end of $\S 3$ we shall also make an explicit correction to the paper of Jehanne, Roblot and Sands [14]. In $\S 4$ we first prove an important reduction of Conjecture 2.1 and then use this to deduce the validity of Conjecture 2.1 in the function field case from a result of Bae in [1] and to show in the number field case that Conjecture 2.1 is a consequence of the 'Strong-Stark Conjecture' that is formulated by Chinburg in [7]. Further, the latter result (Theorem 4.1(v)) combines directly with, for example, a result of Tate to show that Conjecture 2.1 is valid for all (non-trivial irreducible) complex representations with rational valued character and with results of Ritter and Weiss, and of Flach, to show that Conjecture 2.1 is valid for all degree one complex representations of $\operatorname{Gal}(\overline{\mathbb{Q}} / \mathbb{Q})$. In addition, since the Strong-Stark Conjecture has been shown to follow from the 'equivariant Tamagawa number conjecture' formulated by Flach and the present author in [5], Theorem 4.1(v) shows that Conjecture 2.1 follows from the general formalism of Tamagawa number conjectures that originates with Bloch and Kato in [3] and hence provides a much more conceptual approach to the original work of Stark and Chinburg. Indeed, when combined with the main result of [4] (which deals with the abelian case), Theorem 4.1(v) now shows that the equivariant Tamagawa number conjecture underlies all of the 'integral' refinements to Stark's Conjecture of which the present author is aware.

To the best of our knowledge, Tate was the first to ask (in 1999) whether the equivariant Tamagawa number conjecture might provide an 'explanation' for the type of explicit integral refinements of Stark's (non-abelian) Conjecture that had been considered by both Stark and Chinburg. Somewhat belatedly, this article now proves that his intuition was indeed correct.

\section{Algebraic preliminaries}

We fix a finite group $G$, an irreducible finite dimensional complex character $\chi$ of $G$ and a subfield $E$ of $\mathbb{C}$ which is both Galois and of finite degree over $\mathbb{Q}$ and over which $\chi$ can be realised. We let $e_{\bar{\chi}}$ denote the central idempotent $\frac{\chi(1)}{|G|} \sum_{g \in G} \chi(g) g$ of $E[G]$ and fix an indecomposable idempotent $f_{\bar{\chi}}$ of $E[G] e_{\bar{\chi}}$. We write $\mathcal{O}$ for the ring of algebraic integers in $E$, choose a maximal $\mathcal{O}$-order $\mathfrak{M}$ in $E[G]$ which contains $f_{\bar{\chi}}$ and define an $\mathcal{O}$-torsion-free right $\mathcal{O}[G]$-module by setting $T_{\chi}:=f_{\bar{\chi}} \mathfrak{M}$. The associated right $E[G]$-module $V_{\chi}:=E \otimes_{\mathcal{O}} T_{\chi}$ has character $\bar{\chi}$. For any (left) $G$-module $M$ we set $M[\chi]:=T_{\chi} \otimes_{\mathbb{Z}} M$, upon which $G$ acts on the left by $t \otimes_{\mathbb{Z}} m \mapsto t g^{-1} \otimes_{\mathbb{Z}} g(m)$ for each $t \in T_{\chi}, m \in M$ and $g \in G$. Then there is a natural isomorphism of (left) $\mathcal{O}[G]$-modules

$$
M[\chi] \cong \operatorname{Hom}_{\mathcal{O}}\left(T_{\chi}^{*}, \mathcal{O} \otimes_{\mathbb{Z}} M\right)
$$

where $G$ acts in the usual (diagonal) manner on the Hom-set and $T_{\chi}^{*}:=\operatorname{Hom}_{\mathcal{O}}\left(T_{\chi}, \mathcal{O}\right.$ ) is endowed with the natural left $G$-action and hence spans a left $E[G]$-module of character $\bar{\chi}$. 
For any $G$-module $M$, subgroup $J$ of $G$ and integer $i$ we write $H^{i}(J, M)$ for the Tate cohomology in degree $i$ of $M$ with respect to $J$. We also write $M^{J}$, resp. $M_{J}$, for the maximal submodule, resp. maximal quotient module, of $M$ upon which $J$ acts trivially. Then we obtain a left, resp. right, exact functor $M \mapsto M^{\chi}$, resp. $M \mapsto M_{\chi}$, from the category of left $G$-modules to the category of $\mathcal{O}$-modules by setting $M^{\chi}:=M[\chi]^{G}$ and $M_{\chi}:=M[\chi]_{G}=T_{\chi} \otimes_{\mathbb{Z}[G]} M$. For any homomorphism of $G$-modules $f: M \rightarrow N$ we write $f_{\chi}: M_{\chi} \rightarrow N_{\chi}$ and $f^{\chi}: M^{\chi} \rightarrow N^{\chi}$ for the induced homomorphisms of $\mathcal{O}$-modules. The action of $\sum_{g \in G} g$ on $M[\chi]$ induces a homomorphism of $\mathcal{O}$-modules $t(M, \chi): M_{\chi} \rightarrow M^{\chi}$ with kernel $H^{-1}(G, M[\chi])$ and cokernel $H^{0}(G, M[\chi])$. Thus $t(M, \chi)$ is bijective whenever $M$, and hence also $M[\chi]$, is a cohomologically trivial $G$-module. If $M$ is finitely generated and torsion-free, then we shall always regard $M^{\chi}$ as an $\mathcal{O}$-submodule of $\mathcal{O} \otimes_{\mathbb{Z}} M$ by means of the identification described in the following result.

Lemma 1.1. We fix a finitely generated torsion-free $G$-module $M$ and observe that $E[G] \otimes_{\mathbb{Z}} M$ has two commuting left actions of $G$ : the first via left multiplication on $E[G]$ and the second such that each $g$ in $G$ sends $x \otimes_{\mathbb{Z}} m$ to $x g^{-1} \otimes_{\mathbb{Z}} g(m)$ for $x$ in $E[G]$ and $m$ in $M$. We write $\left(E[G] \otimes_{\mathbb{Z}} M\right)^{G, 2}$ for the subset of $E[G] \otimes_{\mathbb{Z}} M$ comprising elements that are invariant under the second action of $G$ and use the first action of $G$ on $E[G] \otimes_{\mathbb{Z}} M$ to regard $\left(E[G] \otimes_{\mathbb{Z}} M\right)^{G, 2}$ as an $E[G]$-module. Then the E-linear map $E[G] \otimes_{\mathbb{Z}} M \rightarrow E \otimes_{\mathbb{Z}} M$ that sends $g \otimes_{\mathbb{Z}} m$ to $g(m)$ for each $g$ in $G$ and $m$ in $M$ restricts to give an isomorphism of $E[G]$-modules $\left(E[G] \otimes_{\mathbb{Z}} M\right)^{G, 2} \cong E \otimes_{\mathbb{Z}} M$. With respect to this isomorphism one has $M^{\chi} \subseteq \chi(1)^{-1} \cdot M^{\chi} \subseteq \mathcal{O} \otimes_{\mathbb{Z}} M$.

Proof. Since $E[G] \otimes_{\mathbb{Z}} M$ is cohomologically-trivial with respect to any action of $G$ the subspace $\left(E[G] \otimes_{\mathbb{Z}} M\right)^{G, 2}$ is equal to the image of $\sum_{g \in G} g$ acting (via the second action of $G)$ on $E[G] \otimes_{\mathbb{Z}} M$ and hence to the $E$-linear span of $\left\{\sum_{g \in G} g^{-1} \otimes_{\mathbb{Z}} g(m): m \in M\right\}$. Using this description it is easy to see that the given map induces an isomorphism of $E[G]$-modules $\left(E[G] \otimes_{\mathbb{Z}} M\right)^{G, 2} \cong E \otimes_{\mathbb{Z}} M$. To prove the second claim we set $N:=$ $\mathcal{O} \otimes_{\mathbb{Z}} M$ and $N^{*}:=\operatorname{Hom}_{\mathcal{O}[G]}(N, \mathcal{O}[G])$. Then, as $N$ is $\mathcal{O}$-torsion-free, it is equal to the set $\left\{x \in E \otimes_{\mathcal{O}} N: \theta(x) \in \mathcal{O}[G]\right.$ for all $\left.\theta \in N^{*}\right\}$. Thus, setting $n:=\chi(1)$, it suffices to prove that $\theta\left(M^{\chi}\right) \subseteq n \cdot \mathcal{O}[G]$ for each $\theta \in N^{*}$. But $\theta\left(M^{\chi}\right) \subseteq \mathbb{Z}[G]^{\chi}$ and, since $\mathbb{Z}[G]$ is cohomologically-trivial and $\mathbb{Z}[G]_{\chi}=T_{\chi}$, one has $\mathbb{Z}[G]^{\chi}=\operatorname{im}(t(\mathbb{Z}[G], \chi))=|G| \cdot T_{\chi}$. Thus it suffices to note that $|G| \cdot T_{\chi}=|G| \cdot f_{\bar{\chi}} \mathfrak{M} \subseteq|G| \cdot e_{\bar{\chi}} \mathfrak{M} \subseteq n \cdot \mathcal{O}[G]$, where the latter inclusion follows from Jacobinski's description in [13] of the central conductor of $\mathfrak{M}$ in $\mathcal{O}[G]$ (see also [9, Th. (27.13)]).

For any $G$-module, resp. $\mathcal{O}$-module, $M$ we write $M_{\text {tor }}$ for the $(\mathbb{Z}$-)torsion submodule of $M$ and set $\bar{M}:=M / M_{\text {tor }}$, which we identify as a submodule of $\mathbb{Q} \otimes_{\mathbb{Z}} M$, resp. $E \otimes_{\mathcal{O}} M$, in the natural way. If $M$ is a $G$-module and $m \in M$, then we write $f_{\bar{\chi}}(m)$ for the image of $f_{\bar{\chi}} \otimes_{\mathbb{Z}[G]} m \in M_{\chi}$ under the natural surjection $M_{\chi} \rightarrow \overline{M_{\chi}}$.

\section{Statement of the conjecture}

We fix a finite Galois extension of global fields $K / k$ and a non-trivial irreducible finite dimensional complex character $\chi$ of $G:=\operatorname{Gal}(K / k)$. For any finite non-empty set of places $S$ of $k$ we write $S(K)$ for the set of places of $K$ which lie above those in $S, Y_{S}$ for the free abelian group on $S(K)$ and $X_{S}$ for the kernel of the homomorphism $Y_{S} \rightarrow \mathbb{Z}$ which sends each element of $S(K)$ to 1 . If $S$ contains the set $S_{\infty}$ 
of all archimedean places of $k$ (in the number field case), then we write $U_{S}$ for the multiplicative subgroup of $K^{\times}$consisting of those elements that are units at all places outside $S$ and $\lambda_{S}: \mathbb{R} \otimes_{\mathbb{Z}} U_{S} \rightarrow \mathbb{R} \otimes_{\mathbb{Z}} X_{S}$ for the isomorphism of $\mathbb{R}[G]$-modules which at each $u \in U_{S}$ satisfies

$$
\lambda_{S}(u)=-\sum_{\pi \in S(K)} \log |u|_{\pi} \cdot \pi
$$

where $|\cdot|_{\pi}$ denotes the normalised absolute value at $\pi$. Now, since $X_{S}$ is torsion-free, the bijectivity of $\lambda_{S}$ combines with $[9, \S 6$, Exer. 6] to imply the existence of injective homomorphisms of $G$-modules of the form $\varphi: X_{S} \rightarrow U_{S}$. For any such $\varphi$ we set

$$
R_{\varphi}^{S}(\chi):=\operatorname{det}_{\mathbb{C}}\left[\left(\mathbb{C} \otimes_{\mathbb{R}} \lambda_{S}\right) \circ\left(\mathbb{C} \otimes_{\mathbb{Z}} \varphi\right) \mid\left(\mathbb{C} \otimes_{E} V_{\chi}\right) \otimes_{\mathbb{Z}[G]} X_{S}\right] \in \mathbb{C}^{\times} .
$$

Then, after taking account of the isomorphism (1), Stark's Conjecture (as interpreted in $[21$, Ch. I, Conj. 5.1]) states that for each $\alpha \in \operatorname{Aut}(\mathbb{C})$ one has

$$
\frac{L_{S}^{*}\left(0, \chi^{\alpha}\right)}{R_{\varphi}^{S}\left(\chi^{\alpha}\right)}=\alpha\left(\frac{L_{S}^{*}(0, \chi)}{R_{\varphi}^{S}(\chi)}\right)
$$

where $L_{S}^{*}(0, \chi)$ denotes the leading non-zero coefficient in the Taylor expansion at $s=0$ of the $S$-truncated Artin $L$-function $L_{S}(s, \chi)$ and $\chi^{\alpha}:=\alpha \circ \chi$.

For each place $v$ in $k$ we fix a place $w$ of $K$ above $v$, write $G_{w}$ for its decomposition subgroup in $G$ and set

$$
r_{S}:=\sum_{v \in S} \operatorname{dim}_{E}\left(V_{\chi}^{G_{w}}\right) .
$$

Then, since $\chi$ is non-trivial, one has

$$
r_{S}=\operatorname{dim}_{E}\left(V_{\chi} \otimes_{\mathbb{Z}[G]} X_{S}\right)=\operatorname{dim}_{E}\left(E \otimes_{\mathcal{O}} X_{S, \chi}\right)
$$

and the function $L_{S}(s, \chi)$ vanishes to order $r_{S}$ at $s=0$ (by [21, Ch. I, Prop. 3.4]). Further, after unwinding the definition of $R_{\varphi}^{S}(\chi)$, one finds that (3) implies

$$
L_{S}^{*}(0, \chi) \cdot \wedge_{E}^{r_{S}}\left(V_{\chi} \otimes_{\mathbb{Z}[G]} X_{S}\right)=\lambda_{S}^{(\chi)}\left(\wedge_{E}^{r_{S}}\left(V_{\chi} \otimes_{\mathbb{Z}[G]} U_{S}\right)\right)
$$

where

$$
\lambda_{S}^{(\chi)}: \mathbb{C} \otimes_{E}\left(\wedge_{E}^{r_{S}}\left(V_{\chi} \otimes_{\mathbb{Z}[G]} U_{S}\right)\right) \stackrel{\sim}{\longrightarrow} \mathbb{C} \otimes_{E}\left(\wedge_{E}^{r_{S}}\left(V_{\chi} \otimes_{\mathbb{Z}[G]} X_{S}\right)\right)
$$

is the isomorphism of $\mathbb{C}$-spaces that is induced by $\lambda_{S}$.

We assume henceforth that the finite (non-empty) set $S$ contains both $S_{\infty}$ (in the number field case) and also all places which ramify in $K / k$. To formulate a conjectural refinement of (4) in this case we find it convenient to avoid complications that arise from the torsion subgroup of $U_{S}$ by using the same method as Tate [21, Ch. IV] and Rubin [18]. Thus, we fix a finite non-empty set of places $T$ of $k$ which is disjoint from $S$ and set

$$
L_{S, T}^{*}(0, \chi):=L_{S}^{*}(0, \chi) \prod_{v \in T} \operatorname{det}_{E}\left(1-\mathrm{N} v \cdot \operatorname{Fr}_{w}^{-1} \mid V_{\chi}\right) \in \mathbb{C}^{\times}
$$

where $\operatorname{Fr}_{w}$ is the Frobenius automorphism of $w$ in $G_{w}$ and $\mathrm{N} v$ is the absolute norm of $v$. We also write $U_{S, T}$ for the (finite index) subgroup of $U_{S}$ consisting of those elements that are congruent to 1 modulo all of the places in $T(K)$. We note that if $U_{S, T}$, and hence $U_{S, T}^{\chi}$, is torsion-free, then the exterior power $\wedge_{\mathcal{O}}^{r_{S}} U_{S, T}^{\chi}$ is also torsion-free and so can be regarded as an $\mathcal{O}$-submodule of $\wedge_{E}^{r_{S}}\left(V_{\chi} \otimes_{\mathbb{Z}[G]} U_{S}\right)$.

We can now state our conjectural refinement of (4). 
Conjecture 2.1. Let $S$ be any finite non-empty set of places of $k$ which contains $S_{\infty}$ and all places which ramify in $K / k$. Let $T$ be any finite non-empty set of places of $k$ that is disjoint from $S$ and such that $U_{S, T}$ is torsion-free. Then (3) is valid for all $\alpha \in \operatorname{Aut}(\mathbb{C})$ and in $\mathbb{C} \otimes_{E}\left(\wedge_{E}^{r_{S}}\left(V_{\chi} \otimes_{\mathbb{Z}[G]} X_{S}\right)\right)$ one has

$$
|G|^{r_{S}} L_{S, T}^{*}(0, \chi) \cdot \wedge_{\mathcal{O}}^{r_{S}} \overline{X_{S, \chi}} \subseteq \operatorname{Fit}_{\mathcal{O}}\left(H^{-1}\left(G, X_{S}[\chi]\right)\right) \cdot \lambda_{S}^{(\chi)}\left(\wedge_{\mathcal{O}}^{r_{S}} U_{S, T}^{\chi}\right) .
$$

We will see (in Proposition 4.8) that it is in fact reasonable to expect that the inclusion of Conjecture 2.1 is an equality for any set $S$ which is 'large enough'.

In $\S 3$ we shall also describe some consequences of Conjecture 2.1 of a much more explicit nature. However, we now end this section by making some clarifying remarks.

Remark 2.2. (The set $T$ ) If $K$ is a (global) function field, then $U_{S, T}$ is torsionfree for any non-empty set $T$. If $K$ is a number field, then $U_{S, T}$ is torsion-free if, for example, not all of the places in $T$ have the same residue characteristic or if $T$ contains a place with residue characteristic greater than $\left|U_{S, \text { tor }}\right|$.

Remark 2.3. (The term $\operatorname{Fit}_{\mathcal{O}}\left(H^{-1}\left(G, X_{S}[\chi]\right)\right)$ ) It is easy to describe an explicit lower bound for the ideal $\operatorname{Fit}_{\mathcal{O}}\left(H^{-1}\left(G, X_{S}[\chi]\right)\right)$ in Conjecture 2.1. Indeed, the exact sequence of $\mathcal{O}[G]$-modules $0 \rightarrow X_{S}[\chi] \rightarrow Y_{S}[\chi] \rightarrow \mathbb{Z}[\chi] \rightarrow 0$ gives rise to an exact sequence of $\mathcal{O}$-modules

$$
H^{-2}(G, \mathbb{Z}[\chi]) \stackrel{\partial}{\rightarrow} H^{-1}\left(G, X_{S}[\chi]\right) \rightarrow H^{-1}\left(G, Y_{S}[\chi]\right) \stackrel{\epsilon}{\rightarrow} H^{-1}(G, \mathbb{Z}[\chi])
$$

and hence to an equality

$$
\begin{aligned}
\operatorname{Fit}_{\mathcal{O}}\left(H^{-1}\left(G, X_{S}[\chi]\right)\right) & =\frac{\operatorname{Fit}_{\mathcal{O}}\left(H^{-1}\left(G, Y_{S}[\chi]\right)\right) \operatorname{Fit}_{\mathcal{O}}(\operatorname{im}(\partial)) \operatorname{Fit}_{\mathcal{O}}(\operatorname{cok}(\epsilon))}{\operatorname{Fit}_{\mathcal{O}}\left(H^{-1}(G, \mathbb{Z}[\chi])\right)} \\
& =\frac{\prod_{v \in S} \operatorname{Fit}_{\mathcal{O}}\left(H^{-1}\left(G_{w}, T_{\chi}\right)\right) \operatorname{Fit}_{\mathcal{O}}(\operatorname{im}(\partial)) \operatorname{Fit}_{\mathcal{O}}(\operatorname{cok}(\epsilon))}{\operatorname{Fit}_{\mathcal{O}}\left(H^{-1}(G, \mathbb{Z}[\chi])\right)}
\end{aligned}
$$

where the last equality follows from the isomorphism $Y_{S}[\chi] \cong \bigoplus_{v \in S} \operatorname{Ind}_{G_{w}}^{G} T_{\chi}$. Also, if $v_{1}$ is any fixed place in $S$ such that $V_{\chi}^{G_{w_{1}}}=0$, for $w_{1}$ the chosen place of $K$ above $v_{1}$, then $T_{\chi}^{G_{w_{1}}}=T_{\chi}^{G}=0$ and so there is a surjective homomorphism of finite $\mathcal{O}$-modules $H^{-1}\left(G_{w_{1}}, T_{\chi}\right)=\left(T_{\chi}\right)_{G_{w_{1}}} \rightarrow\left(T_{\chi}\right)_{G} \cong(\mathbb{Z}[\chi])_{G}=H^{-1}(G, \mathbb{Z}[\chi])$ and hence an inclusion $\operatorname{Fit}_{\mathcal{O}}\left(H^{-1}\left(G_{w_{1}}, T_{\chi}\right)\right) \subseteq \operatorname{Fit}_{\mathcal{O}}\left(H^{-1}(G, \mathbb{Z}[\chi])\right)$. Thus one has

$$
\operatorname{Fit}_{\mathcal{O}}\left(H^{-1}\left(G, X_{S}[\chi]\right)\right) \subseteq \prod_{v \in S \backslash\left\{v_{1}\right\}} \operatorname{Fit}_{\mathcal{O}}\left(H^{-1}\left(G_{w}, T_{\chi}\right)\right) \subseteq \prod_{v} \operatorname{Fit}_{\mathcal{O}}\left(\left(T_{\chi}\right)_{G_{w}}\right)
$$

where the second product is over all places $v$ in $S \backslash\left\{v_{1}\right\}$ with $V_{\chi}^{G_{w}}=0$ and the second inclusion is valid because for each such $v$ one has $H^{-1}\left(G_{w}, T_{\chi}\right)=\left(T_{\chi}\right)_{G_{w}}$. We finally note that if $G_{w}$ is a non-trivial group of prime power order, then Nakayama's lemma implies that $\operatorname{Fit}_{\mathcal{O}}\left(\left(T_{\chi}\right)_{G_{w}}\right)$ is a non-trivial (integral) ideal and hence the term $\operatorname{Fit}_{\mathcal{O}}\left(H^{-1}\left(G, X_{S}[\chi]\right)\right)$ represents a non-trivial integrality constraint on the elements that we discuss in $\S 3$ below.

Remark 2.4. (Special cases) In the following two special cases we are aware of an explicit integral refinement of Stark's conjecture that is actually stronger than Conjecture 2.1. 
(i) The case $r_{S}=0$. In this case (3) implies $L_{S, T}(0, \chi)$ belongs to $E$ and Conjecture 2.1 further predicts that $L_{S, T}(0, \chi)$ belongs to $\operatorname{Fit}_{\mathcal{O}}\left(H^{-1}\left(G, X_{S}[\chi]\right)\right)$. In fact, by using a natural notion of 'non-commutative Fitting invariant', Parker has recently shown that the equivariant Tamagawa number conjecture leads to a stronger restriction on $L_{S, T}(0, \chi)$ in this case (for details see [16]).

(ii) The abelian case. If $\chi$ has degree one, then it factors through the abelianisation $G^{\mathrm{ab}}$ of $G$. Also, if $V_{\chi}^{G_{w}}$ vanishes and $G_{w}^{\mathrm{ab}}$ denotes the image of $G_{w}$ in $G^{\mathrm{ab}}$, then Fit $_{\mathcal{O}}\left(\left(T_{\chi}\right)_{G_{w}}\right)$ is equal to the image of the kernel of the projection map $\mathbb{Z}\left[G^{\text {ab }}\right] \rightarrow$ $\mathbb{Z}\left[G^{\mathrm{ab}} / G_{w}^{\mathrm{ab}}\right]$ under the homomorphism $\mathbb{Z}\left[G^{\mathrm{ab}}\right] \rightarrow \mathcal{O}$ that is induced by $\chi$. By taking this (and Remark 2.3) into account, one can show that in this case the inclusion of Conjecture 2.1 is a consequence of the finer congruence properties of $L_{S, T}^{*}(0, \chi)$ that are discussed in [4]. In particular, in this way one sees that Conjecture 2.1 can be interpreted as a natural non-abelian analogue of the conjecture formulated by Rubin in $[18$, Conj. B].

\section{Stark units}

In this section we shall describe some explicit consequences of Conjecture 2.1. Before stating the first such result we note that if $U_{S, T}$ is torsion-free, then Lemma 1.1 allows us to regard the exterior power $\wedge_{\mathcal{O}}^{r_{S}}\left(\chi(1)^{-1} \cdot U_{S, T}^{\chi}\right)$ as a submodule of $\mathcal{O} \otimes_{\mathbb{Z}} \overline{\wedge_{\mathbb{Z}}^{r_{S}} U_{S, T}}$. We set $\mathfrak{c}_{S}:=\operatorname{Fit}_{\mathcal{O}}\left(H^{-1}\left(G, X_{S}[\chi]\right)\right)$.

Proposition 3.1. Assume that there exists a place $v$ in $S$ for which $V_{\chi}^{G_{w}}$ vanishes (for any fixed place $w$ of $K$ above $v$ ). For each integer $i$ with $1 \leq i \leq r_{S}$ fix a place $w_{i}$ in $S(K)$ and let $\Sigma$ denote the ordered set $\left\{w_{i}: 1 \leq i \leq r_{S}\right\}$. If Conjecture 2.1 is valid, then for any element $d^{\prime}$ of $\mathfrak{c}_{S}^{-1}$ there exists a unique element $u_{\Sigma}\left(d^{\prime}\right)$ of $\wedge_{\mathcal{O}}^{r_{S}}\left(\chi(1)^{-1} \cdot U_{S, T}^{\chi}\right) \subset \mathcal{O} \otimes_{\mathbb{Z}} \overline{\wedge_{\mathbb{Z}}^{r_{S}} U_{S, T}}$ for which

$$
\lambda_{S}^{(\chi)}\left(u_{\Sigma}\left(d^{\prime}\right)\right)=\frac{d^{\prime}|G|^{r_{S}}}{\chi(1)^{r_{S}}} L_{S, T}^{*}(0, \chi) \cdot \wedge_{i=1}^{i=r_{S}} f_{\bar{\chi}}\left(w_{i}\right) .
$$

Proof. The isomorphism $Y_{S}[\chi] \cong \bigoplus_{v \in S} \operatorname{Ind}_{G_{w}}^{G} T_{\chi}$ induces an isomorphism $\left(Y_{S, \chi}\right)_{\text {tor }} \cong$ $\bigoplus_{v \in S}\left(T_{\chi}\right)_{G_{w}, \text { tor }}$. One also has $\left(T_{\chi}\right)_{G_{w} \text {,tor }}=\left(T_{\chi}\right)_{G_{w}}$ whenever $V_{\chi}^{G_{w}}$ vanishes. Thus, the (assumed) existence of a place $v$ in $S$ for which $V_{\chi}^{G_{w}}$ vanishes implies that the natural map $Y_{S} \rightarrow \mathbb{Z}$ induces a surjection $\left(Y_{S, \chi}\right)_{\text {tor }} \rightarrow\left(T_{\chi}\right)_{G}=\mathbb{Z}_{\chi}$. The inclusion $X_{S} \subset Y_{S}$ therefore induces an identification $\overline{X_{S, \chi}}=\overline{Y_{S, \chi}}$ and so the element $\wedge_{i=1}^{i=r_{S}} f_{\bar{\chi}}\left(w_{i}\right)$ belongs to $\wedge_{\mathcal{O}}^{r_{S}} \overline{X_{S, \chi}}$. The existence of an element $u_{\Sigma}\left(d^{\prime}\right)$ of $\wedge_{\mathcal{O}}^{r_{S}}\left(\chi(1)^{-1} \cdot U_{S, T}^{\chi}\right)$ which satisfies (5) thus follows directly from the inclusion of Conjecture 2.1. The uniqueness of $u_{\Sigma}\left(d^{\prime}\right)$ then follows from the injectivity of $\lambda_{S}^{(\chi)}$.

In the rest of this section we describe more explicit versions of Proposition 3.1 in the case that $r_{S}=1$. In this case there exists a unique place $v=v_{1}$ in $S$ with $V_{\chi}^{G_{w_{1}}} \neq 0$ for any fixed place $w_{1}$ of $K$ above $v_{1}$. In addition, the fact that $r_{S}=1$ is the multiplicity of $\chi$ in the $\mathbb{Q}$-rational representation $\mathbb{C} \otimes_{\mathbb{Z}} X_{S}=\mathbb{C} \otimes_{\mathbb{Q}}\left(\mathbb{Q} \otimes_{\mathbb{Z}} X_{S}\right)$ implies that the Schur index of $\chi$ is equal to 1 and so we can take the field $E$ in Proposition 3.1 to be the field of values of $\chi$. We also set $\Gamma:=\operatorname{Gal}(E / \mathbb{Q})$ and write $\mathcal{D}_{E}$ for the different of the extension $E / \mathbb{Q}$. 
For each $\gamma \in \Gamma$ we write $L_{S, T}^{\prime}\left(0, \chi^{\gamma}\right)$ for the value at $s=0$ of the first derivative of $L_{S, T}\left(s, \chi^{\gamma}\right)$.

Proposition 3.2. Assume that $r_{S}=1$ and $|S|>1$. Let $v_{1}$ be the unique place in $S$ with $V_{\chi}^{G_{w_{1}}} \neq 0$ (for any fixed place $w_{1}$ of $K$ above $v_{1}$ ) and set $S_{1}:=S_{\infty} \cup\left\{v_{1}\right\}$. If Conjecture 2.1 is valid, then for any element $d$ of $\mathfrak{c}_{S}^{-1} \mathcal{D}_{E}^{-1}$ there exists an element $u_{w_{1}}(d)$ of $U_{S_{1}, T}$ which at each place $w$ of $K$ satisfies

(6) $-\log \left|u_{w_{1}}(d)\right|_{w}= \begin{cases}0, & \text { if } w \nmid v_{1}, \\ \sum_{\gamma \in \Gamma} \sum_{h \in G_{w_{1}}} \gamma(d) \chi^{\gamma}(g h) L_{S, T}^{\prime}\left(0, \chi^{\gamma}\right), & \text { if } w=g\left(w_{1}\right) \text { for } \\ \text { any } g \text { in } G .\end{cases}$

Proof. We assume throughout this argument that Conjecture 2.1 is valid. We also note that, since both $r_{S}=1$ and $|S|>1$, there exists a place $v$ in $S$ with $V_{\chi}^{G_{w}}=0$ and so Proposition 3.1 applies in this context.

We set $n:=\chi(1)$ and $\operatorname{pr}_{\chi}:=n^{-1}|G| e_{\bar{\chi}}=\sum_{g \in G} \chi(g) g \in \mathcal{O}[G]$ and let $e_{\bar{\chi}}=$ $\sum_{j=1}^{j=n} f_{\bar{\chi}, j}$ be the decomposition of $e_{\bar{\chi}}$ as a sum of indecomposable idempotents in $E[G]$, with $f_{\bar{\chi}, 1}=f_{\bar{\chi}}$. We also fix $d^{\prime} \in \mathfrak{c}_{S}^{-1}$ and $d^{\prime \prime} \in \mathcal{D}_{E}^{-1}$ and set $d:=d^{\prime} d^{\prime \prime}$ (note that, by linearity, it suffices to consider elements $d$ of this form). For each index $j$ as above we then define

$$
\eta(d, \chi, j):=\frac{d|G|}{n} \frac{L_{S, T}^{\prime}(0, \chi)}{R_{\varphi}^{S}(\chi)} \cdot\left(\mathbb{C} \otimes_{\mathcal{O}} \varphi_{\chi}\right)\left(f_{\bar{\chi}, j}\left(w_{1}\right)\right) \in \mathbb{C} \otimes_{\mathcal{O}} U_{S, T}^{\chi}
$$

where, just as in Proposition 3.1, we regard $f_{\bar{\chi}, j}\left(w_{1}\right)$ as an element of $E \otimes_{\mathcal{O}} X_{S, \chi}$.

Now $L_{S, T}^{*}(0, \chi)=L_{S, T}^{\prime}(0, \chi)$ because $r_{S}=1$ and hence, since $f_{\bar{\chi}, 1}\left(w_{1}\right)$ is a nonzero element of the dimension one $\mathbb{C}$-space $\mathbb{C} \otimes_{\mathcal{O}} X_{S, \chi}$, the definition of $R_{\varphi}^{S}(\chi)$ implies that $\lambda_{S}(\eta(d, \chi, 1))=d|G| n^{-1} L_{S, T}^{\prime}(0, \chi) \cdot f_{\bar{\chi}, 1}\left(w_{1}\right)$. Thus $\eta(d, \chi, 1)$ is equal to the element $d^{\prime \prime} \cdot u_{\left\{w_{1}\right\}}\left(d^{\prime}\right)$ of $n^{-1} \mathcal{D}_{E}^{-1} \cdot U_{S_{1}, T}^{\chi}$ that is described by Proposition 3.1. But $V_{\chi}^{G_{w}}=0$ for all $v \in S \backslash\left\{v_{1}\right\}$ so $Y_{S \backslash S_{1}}^{\chi}=0$ and hence the natural exact sequence $0 \rightarrow U_{S_{1}, T} \rightarrow U_{S, T} \rightarrow Y_{S \backslash S_{1}}$ implies $U_{S_{1}, T}^{\chi}=U_{S, T}^{\chi}$. After recalling Lemma 1.1 we therefore deduce that $\eta(d, \chi, 1)$ belongs to $\mathcal{D}_{E}^{-1} \otimes_{\mathbb{Z}} U_{S_{1}, T}$.

Upon replacing $f_{\bar{\chi}}=f_{\bar{\chi}, 1}$ by $f_{\bar{\chi}, j}$ (for any given index $j$ ) in the definition of $T_{\chi}$, the same argument proves that $\eta(d, \chi, j)$ belongs to $\mathcal{D}_{E}^{-1} \otimes_{\mathbb{Z}} U_{S_{1}, T}$ and satisfies $\lambda_{S}(\eta(d, \chi, j))=d|G| n^{-1} L_{S, T}^{\prime}(0, \chi) \cdot f_{\bar{\chi}, j}\left(w_{1}\right)$. The element $\eta(d, \chi):=\sum_{j=1}^{j=n} \eta(d, \chi, j)$ of $\mathcal{D}_{E}^{-1} \otimes_{\mathbb{Z}} U_{S_{1}, T}$ therefore satisfies

$$
d L_{S, T}^{\prime}(0, \chi) \cdot \operatorname{pr}_{\chi}\left(w_{1}\right)=\sum_{j=1}^{j=n} d|G| n^{-1} L_{S, T}^{\prime}(0, \chi) \cdot f_{\bar{\chi}, j}\left(w_{1}\right)=\lambda_{S}(\eta(d, \chi)) .
$$

Further, the assumed validity of (3) combines with the definition of each element $\eta(d, \chi, j)$ to imply that $\eta\left(\gamma(d), \chi^{\gamma}\right)=\gamma(\eta(d, \chi))$ for each $\gamma \in \Gamma$, where on the right hand side we use the natural semi-linear action of $\Gamma$ on $\mathcal{D}_{E}^{-1} \otimes_{\mathbb{Z}} U_{S_{1}, T}$. Thus, if we set $u_{w_{1}}(d):=\sum_{\gamma \in \Gamma} \gamma(\eta(d, \chi))$, then the last displayed formula implies that

$$
\sum_{\gamma \in \Gamma} \gamma(d) L_{S, T}^{\prime}\left(0, \chi^{\gamma}\right) \cdot \operatorname{pr}_{\chi^{\gamma}}\left(w_{1}\right)=\lambda_{S}\left(u_{w_{1}}(d)\right) .
$$


But $\operatorname{Tr}_{E / \mathbb{Q}}\left(\mathcal{D}_{E}^{-1}\right) \subseteq \mathbb{Z}$ and so $u_{w_{1}}(d)$ belongs to $\operatorname{Tr}_{E / \mathbb{Q}}\left(\mathcal{D}_{E}^{-1}\right) \otimes_{\mathbb{Z}} U_{S_{1}, T} \subseteq U_{S_{1}, T}$. It now only remains to note that the definition of $\lambda_{S}$ in (2) implies that (7) is equivalent to the claimed equalities (6).

An elementary exercise in Galois theory shows that (6) implies that the field $K^{\operatorname{ker}(\chi)}$ is generated over $k$ by the $G$-conjugates of $u_{w_{1}}(d)^{N}$ for any large enough integer $N$ (cf. [21, Ch. III, §3.3]). However, to discuss further properties of the conjectural elements $u_{w_{1}}(d)$ we now assume in addition that $k$ is a number field, that $\chi$ has degree two and that $v_{1}$ is (real) archimedean. In this case $G_{w_{1}}$ has order two (since $V_{\chi}^{G_{w_{1}}} \neq V_{\chi}$ ) and we write $\tau$ for its non-trivial element. We also fix an embedding of $K$ in $\mathbb{C}$ that corresponds to $w_{1}$ and use this to regard $K$ as a subfield of $\mathbb{C}$.

Proposition 3.3. We assume that $k$ is a number field, that $r_{S}=1$ and $|S|>1$, that the unique place $v_{1}$ in $S$ with $V_{\chi}^{G_{w_{1}}} \neq 0$ is archimedean and that $\chi$ has degree two. If Conjecture 2.1 is valid, then for each element $d$ of $\mathfrak{c}_{S}^{-1} \mathcal{D}_{E}^{-1}$ the element

$$
\epsilon(d):=\exp \left(-\sum_{\gamma \in \Gamma} \gamma(d) L_{S, T}^{\prime}\left(0, \chi^{\gamma}\right)\right)
$$

is a real unit in $K$ which satisfies all of the following conditions:-

- either $\epsilon(d)$ or $-\epsilon(d)$ is congruent to 1 modulo all of the places in $T(K)$;

- $|\epsilon(d)|_{w}=1$ if $w$ is any place of $K$ which does not lie above $v_{1}$;

- for each $g \in G$ one has

$$
-\log \left|g^{-1}(\epsilon(d))\right|_{w_{1}}=\sum_{\gamma \in \Gamma} \gamma(d)\left(\chi^{\gamma}(g)+\chi^{\gamma}(g \tau)\right) L_{S, T}^{\prime}\left(0, \chi^{\gamma}\right) .
$$

Proof. Since, by assumption, both $\operatorname{dim}_{E}\left(V_{\chi}\right)=2$ and $\operatorname{dim}_{E}\left(V_{\chi}^{G_{w_{1}}}\right)=1$ the set of eigenvalues of the action of $\tau$ on $V_{\chi}$ is equal to $\{-1,+1\}$ and hence $\chi(\tau)=0$. From the equality (6) (with $g$ the identity element of $G$ ) we therefore find that

$$
u_{w_{1}}(d) \cdot \tau\left(u_{w_{1}}(d)\right)=\left|u_{w_{1}}(d)\right|_{w_{1}}=\exp \left(-2 \sum_{\gamma \in \Gamma} \gamma(d) L_{S, T}^{\prime}\left(0, \chi^{\gamma}\right)\right)=\epsilon(d)^{2} .
$$

But from (6) we also know that $\left|\tau\left(u_{w_{1}}(d)\right)\right|_{w}=\left|u_{w_{1}}(d)\right|_{w}$ for all places $w$ of $K$ (indeed, this is clear if $w \nmid v_{1}$ and in the case $w=g\left(w_{1}\right)$ follows easily from the explicit formula given in (6) and the fact that $\chi$ is a class function on $G$ ) and so the element $\left(\epsilon(d) / u_{w_{1}}(d)\right)^{2}=\tau\left(u_{w_{1}}(d)\right) / u_{w_{1}}(d)$ belongs to $U_{S_{1}, T} \cap \operatorname{ker}\left(\lambda_{S}\right)=\left(U_{S_{1}, T}\right)_{\text {tor }}$. Hence, by our choice of $T$, we find that $\left(\epsilon(d) / u_{w_{1}}(d)\right)^{2}=1$ and so $\epsilon(d)= \pm u_{w_{1}}(d)$. From this equality we deduce that $\epsilon(d)$ belongs to $U_{S_{1}}$ and so is a unit in $K$ (since $S_{1}=S_{\infty}$ ). On the other hand, the last displayed equality implies that $\epsilon(d)^{2}$ is a positive real number and hence that $\epsilon(d)$ is itself real. Lastly, the properties of $u_{w_{1}}(d)$ that are described in (6) imply directly that $\epsilon(d)= \pm u_{w_{1}}(d)$ satisfies all of the conditions listed in Proposition 3.3.

Following Proposition 3.3, we now further specialise to the case that $k=\mathbb{Q}$ and $v_{1}$ is archimedean. For simplicity, we also assume that $\mathcal{O}$ is generated as a $\mathbb{Z}$-module by the set $\{\chi(g): g \in G\}$ (as is the case, for example, for all of the examples considered by Fogel in [12] and by Jehanne, Roblot and Sands in [14]). Then [14, Prop. 2.7, Prop. 2.12] shows that, if we replace the condition $d \in \mathfrak{c}_{S}^{-1} \mathcal{D}_{E}^{-1}$ that occurs in Proposition 3.3 by the stronger condition $d \in \mathcal{D}_{E}^{-1}$ and take $S$ equal to the set $S_{0}$ 
consisting of $v_{1}$ and all rational primes which ramify in $K / \mathbb{Q}$, then the question of the existence of real units in $K$ which satisfy the conditions listed in Proposition 3.3 was first considered by Stark in [20]. Also, under our stated assumption about $\mathcal{O}$, the subsequent conjecture [8, Conj. 1] of Chinburg differs from Stark's question only in that $L_{S_{0}}(s, \chi)$ is replaced by the primitive $L$-function $L(s, \chi)$. Thus, modulo these differences (and the occurrence of the auxiliary set of places $T$ ), the factor $\mathfrak{c}_{S_{0}}$ which occurs in Proposition 3.3 predicts that the units that occur in Stark's question and in Chinburg's conjecture should be $N$-th powers of real units in $K^{\times}$where $N$ is the largest integer such that $\mathfrak{c}_{S_{0}} \subseteq N \cdot \mathcal{O}$. We remark that the possibility of such 'extra divisibility' in the Stark-Chinburg Conjecture has already been observed numerically in both [12] and [14] (but see the following remark).

Remark 3.4. (A correction to the paper of Jehanne, Roblot and Sands) The displayed formula which occurs in [14, Rem. 2.10] is incorrect. Indeed, since (in the notation of loc. cit.) the norm $\|-\|$ is attached to a complex place whilst the element $\epsilon(d)$ is real, the left hand side of the given formula should read ' $\epsilon(d)^{2}$ ', rather than ' $\epsilon(d)$ '. Corresponding corrections are then also required in the calculations that are made in $[14, \S 4]$ and hence in the data listed in [14, Table 3]. In particular, this error accounts for the fact that, as explicitly remarked at the beginning of [14, $\S 4.2]$, the authors found that 'the unit $\epsilon(d)$ was a square in $\mathcal{K}$. In fact, in almost all examples, it is actually a fourth power'. Taking account of the necessary corrections, this assertion should have read 'the unit $\epsilon(d)$ belongs to $\mathcal{K}$. In fact, in almost all examples, it is actually a square'. Despite these errors [14, Th. 4.2] remains valid as stated.

\section{The main result}

In this section we prove the following result.

Theorem 4.1. Conjecture 2.1 is valid in each of the following cases:-

(i) $k$ is a global function field;

(ii) $k$ is a number field and $\chi$ is rational valued;

(iii) $k=\mathbb{Q}$ and $\chi$ has degree one;

(iv) $k$ is an imaginary quadratic field of class number one and $\chi$ is a degree one character whose order is divisible only by primes which split completely in $k / \mathbb{Q}$;

(v) $k$ is a number field and the 'Strong-Stark Conjecture' of [7, Conj. 2.2] is valid for $K / k$.

Remark 4.2. (An $A_{4}$ example) In $[6, \S 3]$ it is shown that [7, Conj. 2.2] is a consequence of the 'equivariant Tamagawa number conjecture' of [5, Conj. 4] for the pair $\left(h^{0}(\operatorname{Spec} K), \mathbb{Z}[G]\right)$. Theorem $4.1(\mathrm{v})$ thus combines with a recent result of Navilarekallu in [15, Th. 3] to verify Conjecture 2.1 in an example with $k=\mathbb{Q}, G$ isomorphic to the alternating group of order $12, \chi$ the unique irreducible degree three character of $G$ and $r_{S}=2$.

Our proof of Theorem 4.1 will proceed as follows. We first show that it is enough to prove Conjecture 2.1 for sets $S$ that satisfy a variety of extra hypotheses. We then show that for such sets $S$ the inclusion of Conjecture 2.1 is a consequence of the Strong-Stark Conjecture and finally we deduce Theorem 4.1 from known cases of the latter conjecture. 
4.1. A useful reduction. In this section we use the same notation as in Conjecture 2.1. In addition, for each finite non-empty set of places $\Sigma$ of $k$ that contains $S_{\infty}$ we write $\mathcal{O}_{\Sigma}$ for the subring of $K$ consisting of those elements that are integral at all places outside $\Sigma(K)$.

Proposition 4.3. It suffices to prove Conjecture 2.1 in the case that both of the following conditions are satisfied:-

(i) $\operatorname{Pic}\left(\mathcal{O}_{S}\right)=0$;

(ii) There exists an exact sequence of $G$-modules of the form

$$
0 \rightarrow U_{S, T} \stackrel{\subseteq}{\rightarrow} U_{S} \rightarrow \mathbb{F}_{T}^{\times} \rightarrow 0
$$

where $\mathbb{F}_{T}^{\times}$denotes the direct sum of the multiplicative groups of the residue fields of all places in $T(K)$.

This result follows directly upon combining the next two lemmas.

Lemma 4.4. There exists a finite set $S^{\prime \prime}$ of places of $k$ which do not belong to $S \cup T$, are each totally split in $K / k$ and are such that the conditions ( $i$ ) and (ii) of Proposition 4.3 are satisfied with $S$ replaced by $S^{\prime}:=S \cup S^{\prime \prime}$.

Proof. With $S^{\prime}$ as in the statement of the lemma, there exists a natural exact sequence of $G$-modules of the form

$$
0 \rightarrow U_{S^{\prime}, T} \stackrel{\subseteq}{\longrightarrow} U_{S^{\prime}} \rightarrow \mathbb{F}_{T}^{\times} \rightarrow \operatorname{Pic}\left(\mathcal{O}_{S^{\prime}}\right)_{T} \rightarrow \operatorname{Pic}\left(\mathcal{O}_{S^{\prime}}\right) \rightarrow 0,
$$

where $\operatorname{Pic}\left(\mathcal{O}_{S^{\prime}}\right)_{T}$ is the quotient of the group of fractional ideals of $\mathcal{O}_{S^{\prime}}$ that are prime to $T(K)$ by the subgroup of principal ideals with a generator congruent to 1 modulo all places in $T(K)$ (cf. [18, (1)]). It is therefore enough to show that we may choose $S^{\prime \prime}$ so that $\operatorname{Pic}\left(\mathcal{O}_{S^{\prime}}\right)_{T}=0$. But class field theory identifies $\operatorname{Pic}\left(\mathcal{O}_{S^{\prime}}\right)_{T}$ with $\operatorname{Gal}\left(H_{S^{\prime}, T} / K\right)$ where $H_{S^{\prime}, T}$ is the maximal abelian extension of $K$ which is unramified outside $T(K)$ and is such that all places in $S^{\prime}(K)$, resp. in $T(K)$, are totally split, resp. at most tamely ramified. The existence of a suitable set $S^{\prime \prime}$ thus follows as a consequence of Tchebotarev's Density Theorem.

Lemma 4.5. Let $S^{\prime \prime}$ be any finite set of places of $k$ which do not belong to $S \cup T$ and are each totally split in $K / k$ and set $S^{\prime}:=S \cup S^{\prime \prime}$. If Conjecture 2.1 is valid with $S$ replaced by $S^{\prime}$, then it is also valid as stated.

Proof. An obvious reduction allows us to assume that $S^{\prime \prime}=\{v\}$ for some place $v$ of $k$ which does not belong to $S \cup T$ and splits completely in $K / k$. We fix a place $w$ of $K$ above $v$ and write $F$ for the free abelian group on the set of prime ideals of $\mathcal{O}_{S}$ which lie above $v$. Then $F$ is a free $\mathbb{Z}[G]$-module with basis the prime ideal $\mathfrak{p}$ that corresponds to $w$ and there is an exact sequence of $G$-modules $0 \rightarrow U_{S, T} \stackrel{\subseteq}{\rightarrow} U_{S^{\prime}, T} \stackrel{c}{\rightarrow} F$ in which $c$ is the map which sends each element $x$ of $U_{S^{\prime}, T}$ to the image of $x \mathcal{O}_{S}$ under the natural map from the group of fractional $\mathcal{O}_{S}$-ideals to $F$.

We set $n:=\chi(1)$. If necessary, after replacing $E$ by a larger field, we can assume that $T_{\chi}$ is a free $\mathcal{O}$-module and then fix a basis $\left\{b_{i}: 1 \leq i \leq n\right\}$. For each such index $i$ we then define $\vartheta_{i} \in \operatorname{Hom}_{\mathcal{O}}\left(U_{S^{\prime}, T}^{\chi}, \mathcal{O}\right)$ to be the composite

$$
U_{S^{\prime}, T}^{\chi} \stackrel{c^{\chi}}{\longrightarrow} F^{\chi} \stackrel{|G|^{-1}}{\longrightarrow} F_{\chi} \stackrel{\epsilon}{\longrightarrow} \mathbb{Z}[G]_{\chi}=T_{\chi} \stackrel{b_{i}^{*}}{\longrightarrow} \mathcal{O}
$$


Here we use the fact that (since $F$ is a free $G$-module) $F^{\chi}=\operatorname{im}(t(F, \chi))=|G| \cdot F_{\chi} \subseteq$ $E \otimes_{\mathcal{O}} F_{\chi}$, the isomorphism $\epsilon$ is induced by the map $F \cong \mathbb{Z}[G]$ sending $\mathfrak{p}$ to 1 and $b_{i}^{*}$ is the element of $\operatorname{Hom}_{\mathcal{O}}\left(T_{\chi}, \mathcal{O}\right)$ which satisfies $b_{i}^{*}\left(b_{j}\right)=\delta_{i j}$ for each $j$ with $1 \leq j \leq n$. We also set $r:=r_{S}$ and $r^{\prime}:=r_{S^{\prime}}=r+n$ and write

$$
\Theta: E \otimes_{\mathcal{O}} \wedge_{\mathcal{O}}^{r^{\prime}} U_{S^{\prime}, T}^{\chi} \rightarrow E \otimes_{\mathcal{O}} \wedge_{\mathcal{O}}^{r} U_{S^{\prime}, T}^{\chi}
$$

for the homomorphism that is induced by $\wedge_{i=1}^{i=n} \vartheta_{i}$. Now, since $\operatorname{im}\left(c^{\chi}\right)$ is a torsion-free, and hence projective, $\mathcal{O}$-module, the exact sequence

$$
0 \rightarrow U_{S, T}^{\chi} \stackrel{\subset}{\longrightarrow} U_{S^{\prime}, T}^{\chi} \stackrel{c^{\chi}}{\longrightarrow} F^{\chi}
$$

implies that we can choose a direct sum decomposition of $\mathcal{O}$-modules $U_{S^{\prime}, T}^{\chi}=U_{S, T}^{\chi} \oplus M$ in such a way that $c^{\chi}$ induces an isomorphism $M \cong \operatorname{im}\left(c^{\chi}\right)$. Then

$$
\wedge_{\mathcal{O}}^{r^{\prime}} U_{S^{\prime}, T}^{\chi}=\sum_{i=0}^{i=r^{\prime}} \wedge_{\mathcal{O}}^{i} U_{S, T}^{\chi} \otimes_{\mathcal{O}} \wedge_{\mathcal{O}}^{r^{\prime}-i} M=\wedge_{\mathcal{O}}^{r} U_{S, T}^{\chi} \otimes_{\mathcal{O}} \wedge_{\mathcal{O}}^{n} M,
$$

where the second equality follows from the fact that $U_{S, T}^{\chi}$ and $M$ are projective $\mathcal{O}$ modules of rank $r$ and $n$ respectively. Since $U_{S, T}^{\chi} \subseteq \operatorname{ker}\left(\vartheta_{i}\right)$ for each index $i$ one therefore has

$$
\Theta\left(\wedge_{\mathcal{O}}^{r^{\prime}} U_{S^{\prime}, T}^{\chi}\right)=\left(\wedge_{i=1}^{i=n} \vartheta_{i}\right)\left(\wedge_{\mathcal{O}}^{n} M\right) \cdot \wedge_{\mathcal{O}}^{r} U_{S, T}^{\chi} \subseteq \wedge_{\mathcal{O}}^{r} U_{S, T}^{\chi}
$$

We next set $F^{\prime}:=\mathbb{Z}[G] \cdot w \subset Y_{S^{\prime}}$ and observe that there is a (split) exact sequence of $G$-modules

$$
0 \rightarrow X_{S} \stackrel{\subset}{\rightarrow} X_{S^{\prime}} \stackrel{d}{\rightarrow} F^{\prime} \rightarrow 0
$$

in which $d$ is the composite of the inclusion $X_{S^{\prime}} \subset Y_{S^{\prime}}$ and the natural projection $Y_{S^{\prime}} \rightarrow F^{\prime}$. We also fix an arbitrary element $x$ of $\wedge_{\mathcal{O}}^{r} \overline{X_{S, \chi}}$ and pre-images $\tilde{b}_{j}$ of each element $b_{j}$ under the surjection $\overline{X_{S^{\prime}, \chi}} \stackrel{d_{\chi}}{\longrightarrow} F_{\chi}^{\prime} \stackrel{\epsilon^{\prime}}{\longrightarrow} \mathbb{Z}[G]_{\chi}=T_{\chi}$ where $\epsilon^{\prime}$ is induced by the isomorphism $F^{\prime} \cong \mathbb{Z}[G]$ which sends $w$ to 1 . Then $x \wedge_{\mathcal{O}} \wedge_{j=1}^{j=n} \tilde{b}_{j}$ belongs to $\wedge_{\mathcal{O}}^{r^{\prime}} \overline{X_{S^{\prime}, \chi}}$. Also, since $F^{\prime}$ is a free $G$-module, the last displayed exact sequence induces an isomorphism of $\mathcal{O}$-modules $H^{-1}\left(G, X_{S}[\chi]\right) \cong H^{-1}\left(G, X_{S^{\prime}}[\chi]\right)$ and thus an equality $\operatorname{Fit}_{\mathcal{O}}\left(H^{-1}\left(G, X_{S^{\prime}}[\chi]\right)\right)=\mathfrak{c}_{S}:=\operatorname{Fit}_{\mathcal{O}}\left(H^{-1}\left(G, X_{S}[\chi]\right)\right)$. Hence, by the assumed validity of Conjecture 2.1 for $S^{\prime}$, there exists an element $\xi_{x}$ of $\mathfrak{c}_{S} \cdot \wedge_{\mathcal{O}}^{r^{\prime}} U_{S^{\prime}, T}^{\chi}$ such that

$$
|G|^{r^{\prime}} L_{S^{\prime}, T}^{*}(0, \chi)\left(x \wedge_{\mathcal{O}} \wedge_{j=1}^{j=n} \tilde{b}_{j}\right)=\lambda_{S^{\prime}}^{(\chi)}\left(\xi_{x}\right) .
$$

For each index $i$ with $1 \leq i \leq n$ we now let $\tilde{b}_{i}^{*} \in \operatorname{Hom}_{\mathcal{O}}\left(\overline{X_{S^{\prime}, \chi}}, \mathcal{O}\right)$ denote the composite

$$
\overline{X_{S^{\prime}, \chi}} \stackrel{d_{\chi}}{\longrightarrow} F_{\chi}^{\prime} \stackrel{\epsilon^{\prime}}{\longrightarrow} \mathbb{Z}[G]_{\chi}=T_{\chi} \stackrel{b_{i}^{*}}{\longrightarrow} \mathcal{O} .
$$

Then $\left(\wedge_{i=1}^{i=n} \tilde{b}_{i}^{*}\right)\left(\wedge_{j=1}^{j=n} \tilde{b}_{j}\right)=\left(\wedge_{i=1}^{i=n} b_{i}^{*}\right)\left(\wedge_{j=1}^{j=n} b_{j}\right)=1$. Hence, since $\overline{X_{S, \chi}} \subseteq \operatorname{ker}\left(\tilde{b}_{i}^{*}\right)$ for each index $i$, one has

$$
\begin{aligned}
|G|^{r} L_{S, T}^{*}(0, \chi) \cdot x & =\left(\wedge_{i=1}^{i=n} \tilde{b}_{i}^{*}\right)\left(|G|^{r} L_{S, T}^{*}(0, \chi)\left(x \wedge_{\mathcal{O}} \wedge_{j=1}^{j=n} \tilde{b}_{j}\right)\right) \\
& =\left(\wedge_{i=1}^{i=n} \tilde{b}_{i}^{*}\right)\left((|G| \log (\mathrm{N} w))^{-n}|G|^{r^{\prime}} L_{S^{\prime}, T}^{*}(0, \chi)\left(x \wedge_{\mathcal{O}} \wedge_{j=1}^{j=n} \tilde{b}_{j}\right)\right) \\
& =\left(\wedge_{i=1}^{i=n} \tilde{b}_{i}^{*}\right) \circ \lambda_{S^{\prime}}^{(\chi)}\left((|G| \log (\mathrm{N} w))^{-n} \xi_{x}\right) \\
& =\lambda_{S}^{(\chi)}\left(\Theta\left(\xi_{x}\right)\right),
\end{aligned}
$$


where the second equality is valid because $L_{S^{\prime}, T}^{*}(0, \chi)=(\log (\mathrm{N} w))^{n} L_{S, T}^{*}(0, \chi)$ and the last equality follows from Lemma 4.6 below. Finally we observe that (8) implies that the element $\Theta\left(\xi_{x}\right)$ belongs to $\mathfrak{c}_{S} \cdot \lambda_{S}^{(\chi)}\left(\wedge_{\mathcal{O}}^{r} U_{S, T}^{\chi}\right)$ and so the last displayed formula validates Conjecture 2.1. This completes the proof of Lemma 4.5 and hence also of Proposition 4.3.

Lemma 4.6. On $\mathbb{C} \otimes_{\mathcal{O}} \wedge_{\mathcal{O}}^{r^{\prime}} U_{S^{\prime}, T}^{\chi}$ one has $\left(\wedge_{i=1}^{i=n} \tilde{b}_{i}^{*}\right) \circ \lambda_{S^{\prime}}^{(\chi)}=(|G| \log (\mathrm{N} w))^{n} \cdot \lambda_{S}^{(\chi)} \circ \Theta$.

Proof. We write $\gamma: \mathbb{C} \otimes_{\mathcal{O}} F_{\chi} \cong \mathbb{C} \otimes_{\mathcal{O}} F_{\chi}^{\prime}$ for the isomorphism of $\mathbb{C}$-spaces that is induced by the isomorphism of $G$-modules $F \rightarrow F^{\prime}$ which sends $\mathfrak{p}$ to $w$ and we set $t:=|G| \log (\mathrm{N} w)$. Then the definitions of the maps $\lambda_{S^{\prime}}, c^{\chi}, d_{\chi}, \epsilon$ and $\epsilon^{\prime}$ combine to ensure the commutativity of the following diagram of surjective homomorphisms

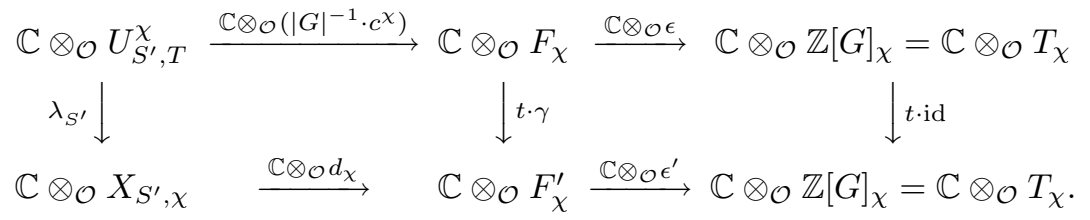

We write $\kappa_{1}$ and $\kappa_{2}$ for the composite homomorphisms given respectively by the upper and lower row of this diagram. It is clear that $\lambda_{S^{\prime}}$ restricts to give a surjection from the kernel $\mathbb{C} \otimes_{\mathcal{O}} U_{S, T}^{\chi}$ of $\mathbb{C} \otimes_{\mathcal{O}}\left(|G|^{-1} \cdot c^{\chi}\right)$ to the kernel $\mathbb{C} \otimes_{\mathcal{O}} X_{S, \chi}$ of $\mathbb{C} \otimes_{\mathcal{O}} d_{\chi}$ and that $\kappa_{2}\left(\tilde{b}_{j}\right)=b_{j}$ for each index $j$. Hence for each $j$ we can fix $u_{j}$ in $\mathbb{C} \otimes_{\mathcal{O}} U_{S^{\prime}, T}^{\chi}$ with both $\kappa_{1}\left(u_{j}\right)=b_{j}$ and $\lambda_{S^{\prime}}\left(u_{j}\right)=t \cdot \tilde{b}_{j}$. Now if $x$ is any non-zero element of $\mathbb{C} \otimes_{\mathcal{O}} \wedge_{\mathcal{O}}^{r} U_{S, T}^{\chi}$, then $x \wedge_{\mathbb{C}} \wedge_{j=1}^{j=n} u_{j}$ is a $\mathbb{C}$-basis of $\mathbb{C} \otimes_{\mathcal{O}} \wedge_{\mathcal{O}}^{r^{\prime}} U_{S^{\prime}, T}^{\chi}$. The claimed equality of homomorphisms is thus valid because

$$
\begin{aligned}
\left(\left(\wedge_{i=1}^{i=n} \tilde{b}_{i}^{*}\right) \circ \lambda_{S^{\prime}}^{(\chi)}\right)\left(x \wedge_{\mathbb{C}} \wedge_{j=1}^{j=n} u_{j}\right) & =\left(\wedge_{i=1}^{i=n} \tilde{b}_{i}^{*}\right)\left(\lambda_{S}^{(\chi)}(x) \wedge_{\mathbb{C}} \wedge_{j=1}^{j=n} \lambda_{S^{\prime}}\left(u_{j}\right)\right) \\
& =t^{n} \cdot \lambda_{S}^{(\chi)}(x) \wedge_{\mathbb{C}}\left(\wedge_{i=1}^{i=n} \tilde{b}_{i}^{*}\right)\left(\wedge_{j=1}^{j=n} \tilde{b}_{j}\right) \\
& =t^{n} \cdot \lambda_{S}^{(\chi)}(x) \wedge_{\mathbb{C}}\left(\wedge_{i=1}^{i=n} b_{i}^{*}\right)\left(\wedge_{j=1}^{j=n} \kappa_{1}\left(u_{j}\right)\right) \\
& =t^{n} \cdot \lambda_{S}^{(\chi)}(x) \wedge_{\mathbb{C}}\left(\wedge_{i=1}^{i=n} \vartheta_{i}\right)\left(\wedge_{j=1}^{j=n} u_{j}\right) \\
& =t^{n} \cdot\left(\lambda_{S}^{(\chi)} \circ \Theta\right)\left(x \wedge_{\mathbb{C}} \wedge_{j=1}^{j=n} u_{j}\right) .
\end{aligned}
$$

Here we use the equalities $\mathbb{C} \otimes_{\mathcal{O}} \vartheta_{i}=\left(\mathbb{C} \otimes_{\mathcal{O}} b_{i}^{*}\right) \circ \kappa_{1}$ and the fact that $\left(\wedge_{i=1}^{i=n} \tilde{b}_{i}^{*}\right)\left(\wedge_{j=1}^{j=n} \tilde{b}_{j}\right)$ and $\left(\wedge_{i=1}^{i=n} b_{i}^{*}\right)\left(\wedge_{j=1}^{j=n} \kappa_{1}\left(u_{j}\right)\right)=\left(\wedge_{i=1}^{i=n} b_{i}^{*}\right)\left(\wedge_{j=1}^{j=n} b_{j}\right)$ are both equal to 1 .

4.2. The proof of Theorem 4.1. We assume henceforth that $S$ is as in Proposition 4.3. Then the validity of $(3)$ for each $\alpha \in \operatorname{Aut}(\mathbb{C})$ implies that $L_{S}^{*}(0, \chi) / R_{\varphi}^{S}(\chi)$ belongs to $E$. Further, in this case the 'Strong-Stark Conjecture' of Chinburg predicts that

$$
\frac{L_{S}^{*}(0, \chi)}{R_{\varphi}^{S}(\chi)} \mathcal{O}=q\left(\psi_{\chi}\right)^{-1},
$$

where $\psi_{\chi}$ denotes the composite homomorphism of $\mathcal{O}$-modules

$$
X_{S, \chi} \stackrel{\varphi_{\chi}}{\longrightarrow} U_{S, \chi} \stackrel{t\left(U_{S}, \chi\right)}{\longrightarrow} U_{S}^{\chi}
$$


Here we have used the following general notation: for any homomorphism of finitely generated $\mathcal{O}$-modules $f: M \rightarrow N$ which has both finite kernel and finite cokernel we define a fractional $\mathcal{O}$-ideal by setting

$$
q(f):=\frac{\operatorname{Fit}_{\mathcal{O}}(\operatorname{cok}(f))}{\operatorname{Fit}_{\mathcal{O}}(\operatorname{ker}(f))} .
$$

Remark 4.7. (The Strong Stark Conjecture) After taking account of the isomorphism (1), it is straightforward to check that if $k$ is a number field, resp. a global function field, then (9) is equivalent to the conjecture formulated by Chinburg in [7, Conj. $2.2]$, resp. to the conjecture discussed by Bae in $[1, \S 3.1]$ as a natural analogue of $[7$, Conj. 2.2].

Proposition 4.8. Let $S$ be as in Proposition 4.3. If (9) is valid, then

$$
|G|^{r_{S}} L_{S, T}^{*}(0, \chi) \cdot \wedge_{\mathcal{O}}^{r_{S}} \overline{X_{S, \chi}}=\operatorname{Fit}_{\mathcal{O}}\left(H^{-1}\left(G, X_{S}[\chi]\right)\right) \cdot \lambda_{S}^{(\chi)}\left(\wedge_{\mathcal{O}}^{r_{S}} U_{S, T}^{\chi}\right)
$$

and so the inclusion of Conjecture 2.1 is also valid in this case.

Proof. We set $r:=r_{S}, X:=X_{S}, U:=U_{S}$ and $U_{T}:=U_{S, T}$. We also write $i_{T}: U_{T}^{\chi} \rightarrow$ $U^{\chi}$ for the homomorphism that is induced by the inclusion $U_{T} \rightarrow U$.

Then from Lemma 4.9 below it follows that $L_{S, T}^{*}(0, \chi) \cdot \wedge_{\mathcal{O}}^{r} \overline{X_{\chi}}$ is equal to

$$
\begin{aligned}
L_{S}^{*}(0, \chi) \cdot q\left(i_{T}\right) \wedge_{\mathcal{O}}^{r} \overline{X_{\chi}} & =R_{\varphi}^{S}(\chi) \frac{q\left(i_{T}\right)}{q\left(\psi_{\chi}\right)} \wedge_{\mathcal{O}}^{r} \overline{X_{\chi}} \\
& =\frac{q\left(i_{T}\right)}{q\left(\psi_{\chi}\right)}\left(\lambda_{S}^{(\chi)} \circ \wedge_{\mathbb{C}}^{r}\left(\mathbb{C} \otimes_{\mathcal{O}} \varphi_{\chi}\right)\right)\left(\wedge_{\mathcal{O}}^{r} \overline{X_{\chi}}\right) \\
& =\frac{q\left(i_{T}\right) q\left(\varphi_{\chi}\right) \operatorname{Fit}_{\mathcal{O}}\left(X_{\chi, \text { tor }}\right)}{q\left(\psi_{\chi}\right) \operatorname{Fit}_{\mathcal{O}}\left(U_{\chi, \text { tor }}\right)} \lambda_{S}^{(\chi)}\left(\wedge_{\mathcal{O}}^{r} \overline{U_{\chi}}\right) \\
& =\frac{q\left(i_{T}\right) \operatorname{Fit}_{\mathcal{O}}\left(X_{\chi, \text { tor }}\right)}{q(t(U, \chi)) \operatorname{Fit}_{\mathcal{O}}\left(U_{\chi, \text { tor }}\right)} \lambda_{S}^{(\chi)}\left(\wedge_{\mathcal{O}}^{r} \overline{U_{\chi}}\right) \\
& =\frac{q\left(i_{T}\right) \operatorname{Fit}_{\mathcal{O}}\left(X_{\chi, \text { tor }}\right)}{|G|^{r} \operatorname{Fit}_{\mathcal{O}}\left(U_{\text {tor }}^{\chi}\right)} \lambda_{S}^{(\chi)}\left(\wedge_{\mathcal{O}}^{r} \overline{U^{\chi}}\right) \\
& =|G|^{-r} \operatorname{Fit}_{\mathcal{O}}\left(X_{\chi, \text { tor }}\right) \lambda_{S}^{(\chi)}\left(\wedge_{\mathcal{O}}^{r} U_{T}^{\chi}\right) .
\end{aligned}
$$

Indeed, the first equality here follows from (9), the second from the definition of $R_{\varphi}^{S}(\chi)$ and the fourth from the equality $q\left(\psi_{\chi}\right)=q(t(U, \chi)) q\left(\varphi_{\chi}\right)$ which is a consequence of the kernel-cokernel sequence of the composite $\psi_{\chi}=t(U, \chi) \circ \varphi_{\chi}$. The third, fifth and sixth equalities in (10) follow by applying Lemma 4.10 below with $f$ equal to $\varphi_{\chi}: X_{\chi} \rightarrow U_{\chi}, t(U, \chi): U_{\chi} \rightarrow U^{\chi}$ and $i_{T}: U_{T}^{\chi} \rightarrow U^{\chi}$ respectively.

To deduce the equality of Proposition 4.8 from (10) it is enough to show that $X_{\chi, \text { tor }}=H^{-1}(G, X[\chi])$. But, since $X^{\chi} \subseteq X[\chi]$ is torsion-free, this follows directly from the exact sequence $0 \rightarrow H^{-1}(G, X[\chi]) \rightarrow X_{\chi} \stackrel{t(X, \chi)}{\longrightarrow} X^{\chi}$. This completes the proof of Proposition 4.8 .

Lemma 4.9. $L_{S, T}^{*}(0, \chi) \cdot \mathcal{O}=L_{S}^{*}(0, \chi) \cdot q\left(i_{T}\right) \subset \mathbb{C}$. 
Proof. For each place $v$ in $T$ we fix a place $w$ of $K$ above $v$ and write $\mathbb{F}_{w}$ for the residue field of $w$. Then, since $v$ is unramified in $K / k$ there is an exact sequence of $G_{w}$-modules

$$
0 \rightarrow \mathbb{Z}\left[G_{w}\right] \stackrel{\cdot\left(1-\mathrm{N} v \cdot \mathrm{Fr}_{w}^{-1}\right)}{\longrightarrow} \mathbb{Z}\left[G_{w}\right] \rightarrow \mathbb{F}_{w}^{\times} \rightarrow 0
$$

in which the third arrow sends the identity element of $G_{w}$ to any choice of generator of the (cyclic) group $\mathbb{F}_{w}^{\times}$. Inducing this sequence from $G_{w}$ to $G$ and then summing over all places in $T$ gives an exact sequence of $G$-modules

$$
0 \rightarrow \bigoplus_{v \in T} \mathbb{Z}[G] \stackrel{\left(\rho_{w}\right)_{v}}{\longrightarrow} \bigoplus_{v \in T} \mathbb{Z}[G] \rightarrow \mathbb{F}_{T}^{\times} \rightarrow 0
$$

in which $\rho_{w}$ denotes the endomorphism of $\mathbb{Z}[G]$ given by $x \mapsto x\left(1-\mathrm{N} v \cdot \mathrm{Fr}_{w}^{-1}\right)$. This sequence implies that $\mathbb{F}_{T}^{\times}$, and hence also $\mathbb{F}_{T}^{\times}[\chi]$, is a cohomologically-trivial $G$-module and this fact combines with the exact sequence

$$
0 \rightarrow U_{T}[\chi] \rightarrow U[\chi] \rightarrow \mathbb{F}_{T}^{\times}[\chi] \rightarrow 0
$$

coming from Proposition 4.3(ii) to imply that the induced map $H^{1}\left(G, U_{T}[\chi]\right) \rightarrow$ $H^{1}(G, U[\chi])$ is bijective. The last displayed sequence therefore gives rise to an exact sequence of $\mathcal{O}$-modules $0 \rightarrow U_{T}^{\chi} \stackrel{i_{T}}{\longrightarrow} U^{\chi} \rightarrow\left(\mathbb{F}_{T}^{\times}\right)^{\chi} \rightarrow 0$. Thus, since $U_{T, \text { tor }}^{\chi}=0$ (by our choice of $T)$, one has $q\left(i_{T}\right)=\operatorname{Fit}_{\mathcal{O}}\left(\left(\mathbb{F}_{T}^{\times}\right)^{\chi}\right)$.

Now the sequence (11) also induces an exact sequence of $\mathcal{O}$-modules

$$
0 \rightarrow \bigoplus_{v \in T} \mathbb{Z}[G]^{\chi} \stackrel{\left(\rho_{w}^{\chi}\right)_{v}}{\longrightarrow} \bigoplus_{v \in T} \mathbb{Z}[G]^{\chi} \rightarrow\left(\mathbb{F}_{T}^{\times}\right)^{\chi} \rightarrow 0
$$

and so, since $\mathbb{Z}[G]^{\chi}$ is a projective $\mathcal{O}$-module, $\operatorname{Fit}_{\mathcal{O}}\left(\left(\mathbb{F}_{T}^{\times}\right)^{\chi}\right)=\left(\prod_{v \in T} \operatorname{det}_{E}\left(\rho_{w}^{\chi}\right)\right) \cdot \mathcal{O}$. But, with respect to the natural identification of $\mathbb{Z}[G]^{\chi}$ with $T_{\chi}$, the endomorphism $\rho_{w}^{\chi}$ corresponds to the action of $1-\mathrm{N} v \cdot \mathrm{Fr}_{w}^{-1}$ on $T_{\chi}$. The ideal $q\left(i_{T}\right)=\operatorname{Fit}_{\mathcal{O}}\left(\left(\mathbb{F}_{T}^{\times}\right)^{\chi}\right)$ is therefore equal to

$$
\left(\prod_{v \in T} \operatorname{det}_{E}\left(\rho_{w}^{\chi}\right)\right) \cdot \mathcal{O}=\left(\prod_{v \in T} \operatorname{det}_{E}\left(1-\mathrm{N} v \cdot \mathrm{Fr}_{w}^{-1} \mid V_{\chi}\right)\right) \cdot \mathcal{O}=\frac{L_{S, T}^{*}(0, \chi)}{L_{S}^{*}(0, \chi)} \cdot \mathcal{O},
$$

as required.

Lemma 4.10. If $f: M \rightarrow N$ is any homomorphism of finitely generated $\mathcal{O}$-modules which has both finite kernel and finite cokernel, then

$$
\wedge_{E}^{d}\left(E \otimes_{\mathcal{O}} f\right)\left(\wedge_{\mathcal{O}}^{d} \bar{M}\right)=q(f) \frac{\operatorname{Fit}_{\mathcal{O}}\left(M_{\text {tor }}\right)}{\operatorname{Fit}_{\mathcal{O}}\left(N_{\text {tor }}\right)} \wedge_{\mathcal{O}}^{d} \bar{N}
$$

with $d:=\operatorname{dim}_{E}\left(E \otimes_{\mathcal{O}} M\right)$. 
Proof. We consider the following exact commutative diagram

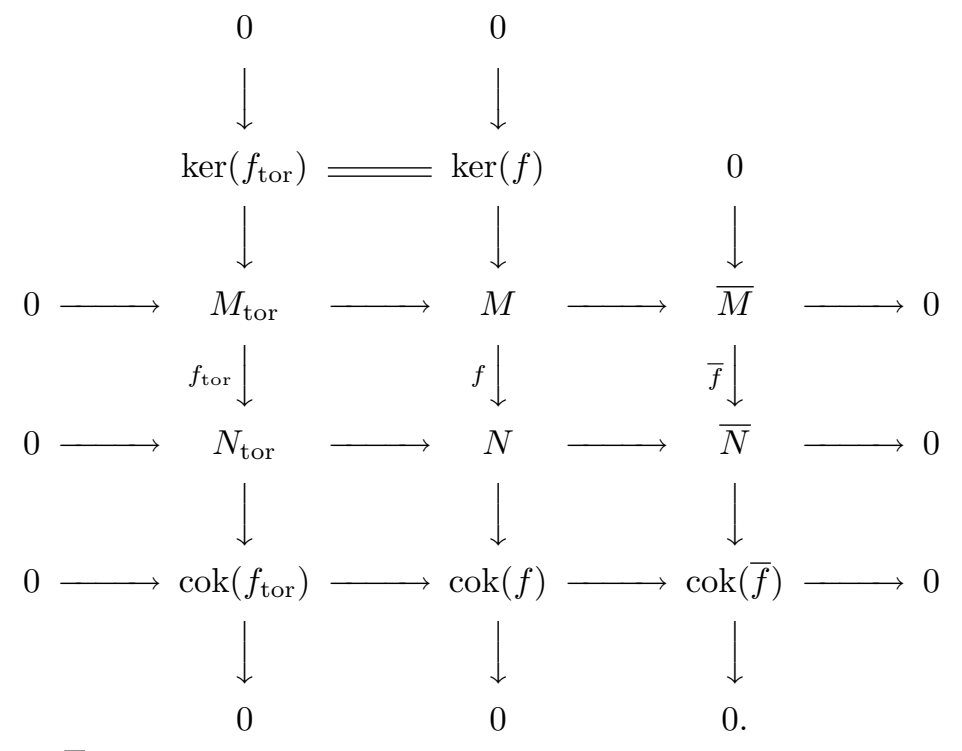

Here $f_{\text {tor }}$ and $\bar{f}$ denote the homomorphisms that are induced by the given map $f$; the equality $\operatorname{ker}\left(f_{\text {tor }}\right)=\operatorname{ker}(f)$ and the injectivity of $\bar{f}$ both follow from the assumption that $\operatorname{ker}(f)$ is finite and the exactness of the bottom row follows by an application of the Snake lemma. Now $\bar{M}$, and hence also $\bar{N}$ since $\operatorname{cok}(\bar{f})$ is finite, is a projective $\mathcal{O}$-modules of rank $d$ and so the definition of Fit $_{\mathcal{O}}(\operatorname{cok}(\bar{f}))$ implies that $\wedge_{E}^{d}\left(E \otimes_{\mathcal{O}} f\right)\left(\wedge_{\mathcal{O}}^{d} \bar{M}\right)=$ Fit $_{\mathcal{O}}(\operatorname{cok}(\bar{f})) \cdot \wedge_{\mathcal{O}}^{d} \bar{N}$. On the other hand, the exactness of the bottom row and left hand column of the above diagram combines with the multiplicativity of Fitting ideals on exact sequences of finite $\mathcal{O}$-modules and the definition of $q(f)$ to imply that $\operatorname{Fit}_{\mathcal{O}}(\operatorname{cok}(\bar{f}))=$ Fit $_{\mathcal{O}}(\operatorname{cok}(f))$ Fit $_{\mathcal{O}}\left(\operatorname{cok}\left(f_{\text {tor }}\right)\right)^{-1}=$ $q(f) \operatorname{Fit}_{\mathcal{O}}\left(M_{\text {tor }}\right) \operatorname{Fit}_{\mathcal{O}}\left(N_{\text {tor }}\right)^{-1}$. The claimed equality is thus clear.

Proposition 4.8 shows that a stronger version of Conjecture 2.1 (in which the inclusion is replaced by an equality) is a consequence of the equality (9) for any set $S$ as in Proposition 4.3. It also shows that Theorem 4.1(v) follows directly from Remark 4.7 and the result of Proposition 4.3. Further, all of the remaining claims of Theorem 4.1 now follow by combining the same observation with the fact that the equality (9) is known to be valid in each of the following cases:-

- $k$ is a global function field: in this case (9) was proved by Bae in [1, Th. 3.5.4];

- $k$ is a number field and $\chi$ is rational valued: in this case (9) was proved by Tate in [21, Ch. II, Th. 6.8];

- $k=\mathbb{Q}$ and $\chi$ has degree one: if 2 is unramified in $K / \mathbb{Q}$, then (9) was proved by Ritter and Weiss in [17] and the remaining 2-primary difficulties were subsequently resolved by Flach (indeed this follows by combining the first assertion of Remark 4.2 with the results of [11, Th. 5.1, Th. 7.1(c)]);

- $k$ is an imaginary quadratic field of class number one and $\chi$ is a degree one character whose order is divisible only by primes which split completely in $k / \mathbb{Q}$ : in this case (9) follows from (the first assertion of Remark 4.2 and) the result of Bley in [2, Th. 4.2]. 


\section{Acknowledgements}

It is a pleasure to thank Cristian Popescu, Jonathan Sands and the referee for some very helpful remarks.

\section{References}

[1] S. Bae, On the conjectures of Lichtenbaum and Chinburg over function fields, Math. Ann. 285 (1989) 417-445.

[2] W. Bley, On the equivariant Tamagawa number conjecture for abelian extensions of a quadratic imaginary field, Documenta Math. 11 (2006) 73-118.

[3] S. Bloch and K. Kato, L-functions and Tamagawa numbers of motives, Prog. in Math. 86, Vol. I, Birkhäuser, (1990) 333-400.

[4] D. Burns, Congruences between derivatives of abelian L-functions at $s=0$, Inventiones Math. 169 (2007) 451-499.

[5] D. Burns and M. Flach, Tamagawa numbers for motives with (non-commutative) coefficients, Documenta Math. 6 (2001) 501-570.

[6] D. Burns and M. Flach, Tamagawa numbers for motives with (non-commutative) coefficients II, Amer. J. Math. 125 (2003) 475-512.

[7] T. Chinburg, On the Galois structure of algebraic integers and S-units, Inventiones Math. 74 (1983) 321-349.

[8] T. Chinburg, Stark's Conjecture for L-Functions with First-Order Zeroes at $s=0$, Advances in Math. 48 (1983) 82-113.

[9] C. W. Curtis and I. Reiner, Methods of Representation Theory, Vol. I, John Wiley and Sons, New York, 1987.

[10] D. S. Dummit, Computations related to Stark's conjecture, Contemp. Math. 358, 37-54, Amer. Math. Soc., 2004.

[11] M. Flach, The equivariant Tamagawa number conjecture - A survey, Contemp. Math. 358, 79-126, Amer. Math. Soc., 2004.

[12] K. Fogel, Stark's Conjecture for Octahedral extensions, PhD Thesis, University of Texas at Austin, 1998.

[13] H. Jacobinski, On extensions of lattices, Michigan Math. J. 13 (1966) 471-475.

[14] A. Jehanne, X-F. Roblot, and J. Sands, Numerical Verification of the Stark-Chinburg Conjecture for Some Icosahedral Representations, Experimental Math. 12 (2003) No. 4, 419-432.

[15] T. Navilarekallu, On the equivariant Tamagawa number conjecture for $A_{4}$-extensions of number fields, J. Number Theory 121 (2006) 67-89.

[16] A. H. Parker, Equivariant Tamagawa numbers and non-commutative Fitting invariants, PhD Thesis, King's College London, 2007.

[17] J. Ritter and A. Weiss, Cohomology of units and L-values at zero, J. Amer. Math. Soc. 10 (1997) 513-552.

[18] K. Rubin, A Stark Conjecture 'over $\mathbb{Z}$ ' for abelian L-functions with multiple zeros, Ann. Inst. Fourier 46 (1996) 33-62.

[19] H. M. Stark, Values of L-functions at $s=1, I V$ : First derivatives at $s=0$, Advances in Math. 35 (1980) 197-235.

[20] H. M. Stark, Derivatives of L-series at $s=0$, in 'Automorphic forms, representation theory and arithmetic (Bombay, 1979)', pp. 261-273, Tata Inst. Fund. Res. Studies in Math., 10, Tata Inst. Fund. Res., Bombay, 1981.

[21] J. Tate, Les Conjectures de Stark sur les Fonctions $L$ d'Artin en $s=0$ (notes par D. Bernardi et N. Schappacher), Progress in Math., 47, Birkhäuser, Boston, 1984.

King's College London, Department of Mathematics, Strand, London WC2R 2LS, UK

E-mail address: david.burns@kcl.ac.uk 